The Astrophysical Journal, 669:1209-1219, 2007 November 10

(C) 2007. The American Astronomical Society. All rights reserved. Printed in U.S.A.

\title{
THE ORBITS OF THE QUADRUPLE STAR SYSTEM 88 TAURI A FROM PHASES DIFFERENTIAL ASTROMETRY AND RADIAL VELOCITY
}

\author{
Benjamin F. Lane, ${ }^{1}$ Matthew W. Muterspaugh, ${ }^{2,3}$ Francis C. Fekel ${ }^{4}$ Michael Williamson, ${ }^{4}$ Stanley Browne, ${ }^{2}$ \\ Maciej Konacki, ${ }^{5}$ Bernard F. Burke, ${ }^{1}$ M. M. Colavita, ${ }^{6}$ S. R. Kulkarni, ${ }^{7}$ and M. Shao ${ }^{6}$ \\ Received 2007 May 22; accepted 2007 June 13
}

\begin{abstract}
We have used high-precision differential astrometry from the Palomar High-precision Astrometric Search for Exoplanet Systems (PHASES) project and radial velocity measurements covering a time span of 20 years to determine the orbital parameters of the 88 Tau A system. 88 Tau is a complex hierarchical multiple system comprising a total of six stars; we have studied the brightest four, consisting of two short-period pairs orbiting each other with an $\sim 18$ yr period. We present the first orbital solution for one of the short-period pairs, and determine the masses of the components and distance to the system to the level of a few percent. In addition, our astrometric measurements allow us to make the first determination of the mutual inclinations of the orbits. We find that the subsystems are not coplanar.
\end{abstract}

Subject headings: stars: individual (88 Tauri) — techniques: interferometric

\section{INTRODUCTION}

88 Tau (HD 29140, HR 1458, HIP 21402) is a bright $\left(m_{V}=\right.$ 4.25, $m_{K}=3.69 \pm 0.25$; Skrutskie et al. 2006), nearby ( $\left.\sim 50 \mathrm{pc}\right)$ hierarchical sextuple stellar system (Tokovinin 1997). The A component contains a pair of systems (designated $\mathrm{Aa}$ and $\mathrm{Ab}$ ) in an $\sim 18$ yr (Balega et al. 1999) orbit that has been resolved by speckle interferometry (McAlister et al. 1987). The Aa component is a known spectroscopic binary system $(P \sim 3.57$ days), with a composite spectral type of A5m (Cowley et al. 1969). In previous work it had been noted (Burkhart \& Coupry 1988) that the A system is likely complex, with possibly as many as five components. Balega et al. (1999) noted a discrepancy between the total estimated mass of this system based on photometry and spectral types, and the total mass derived from the visual orbit and Hipparcos parallax. In this work we have determined that, like the Aa component, the $\mathrm{Ab}$ component is a double-lined binary; this newly resolved binary has a period of 7.89 days. Finally, there is a common-propermotion companion, labeled $\mathrm{B}$, located $\sim 69^{\prime \prime}$ away from the A system; it, too, is known to be a binary ( Tokovinin \& Gorynya 2001). For clarity we provide a schematic of this complex system in Figure 1.

There are several reasons why multiple stellar systems such as 88 Tau merit attention: first, binary orbits make it possible to measure accurate stellar masses and distances, while the larger number of presumably coeval stars allows one to impose the additional constraint that any given model must accurately match all of the stars. This approach has proven particularly fruitful when applied to another famous hierarchical sextuple system: Castor (Torres \&

\footnotetext{
1 Kavli Institute for Astrophysics and Space Research and Department of Physics, Massachusetts Institute of Technology, 70 Vassar Street, Cambridge, MA 02139.

2 Space Sciences Laboratory, University of California, 7 Gauss Way, Berkeley, CA 94720-7450.

3 Townes Fellow.

4 Center of Excellence in Information Systems, Tennessee State University, 3500 John A. Merritt Boulevard, Box 9501, Nashville, TN 37209.

5 Nicolaus Copernicus Astronomical Center, Polish Academy of Sciences, Rabianska 8, 87-100 Torun, Poland.

6 Jet Propulsion Laboratory, California Institute of Technology, 4800 Oak Grove Drive, Pasadena, CA 91109.

7 Division of Physics, Mathematics, and Astronomy, 105-24, California Institute of Technology, Pasadena, CA 91125.
}

Ribas 2002). Second, as outlined in Sterzik \& Tokovinin (2002) the relative orientations of the orbital angular momenta allow one to constrain the properties of the cloud from which the stars are thought to have formed, as well as the subsequent dynamical decay process. Despite their value, observational problems have limited the number of triple or higher order systems with accurately measured orbits to fewer than 10. Given their hierarchical nature it is often the case that either the close system is unresolvable or the outer system has an impractically long orbital period.

With the advent of long-baseline stellar interferometry, and more recently phase-referenced long-baseline interferometric astrometry (Lane \& Muterspaugh 2004) capable of 10-20 $\mu$ as astrometric precision between pairs of stars with separations in the range $0.05^{\prime \prime}-1^{\prime \prime}$, it has become possible to resolve the orbital motion of several interesting multiple systems (Muterspaugh et al. 2006a, 2006b). Here we report on astrometric and radial velocity measurements of the 88 Tau A system, which allow us to constrain the orbits of the 3.57 day, 7.89 day, and $18 \mathrm{yr}$ components with improved precision, and for the first time provide a relative orientation of the orbits as well as component masses.

Astrometric measurements were made with the Palomar Testbed Interferometer (Colavita et al. 1999) as part of the Palomar Highprecision Astrometric Search for Exoplanet Systems (PHASES) program (Muterspaugh et al. 2006c). The Palomar Testbed Interferometer is located on Palomar Mountain near San Diego, California. It was developed by the Jet Propulsion Laboratory, California Institute of Technology for NASA as a testbed for interferometric techniques applicable to the Keck Interferometer and the Space Interferometry Mission (SIM). It operates in the $J(1.2 \mu \mathrm{m}), H(1.6 \mu \mathrm{m})$, and $K(2.2 \mu \mathrm{m})$ bands and combines starlight from two out of three available $40 \mathrm{~cm}$ apertures. The apertures form a triangle with 86 and $110 \mathrm{~m}$ baselines.

\section{OBSERVATIONS AND MODELS}

\subsection{PHASES Astrometry}

88 Tau A was successfully observed with PTI on 29 nights in 2004-2007 with the use of the phase-referenced fringe-scanning mode (Lane \& Muterspaugh 2004) developed for high-precision astrometry; the data were reduced with the algorithms described therein, as well as with the modifications described in Muterspaugh et al. (2005). 


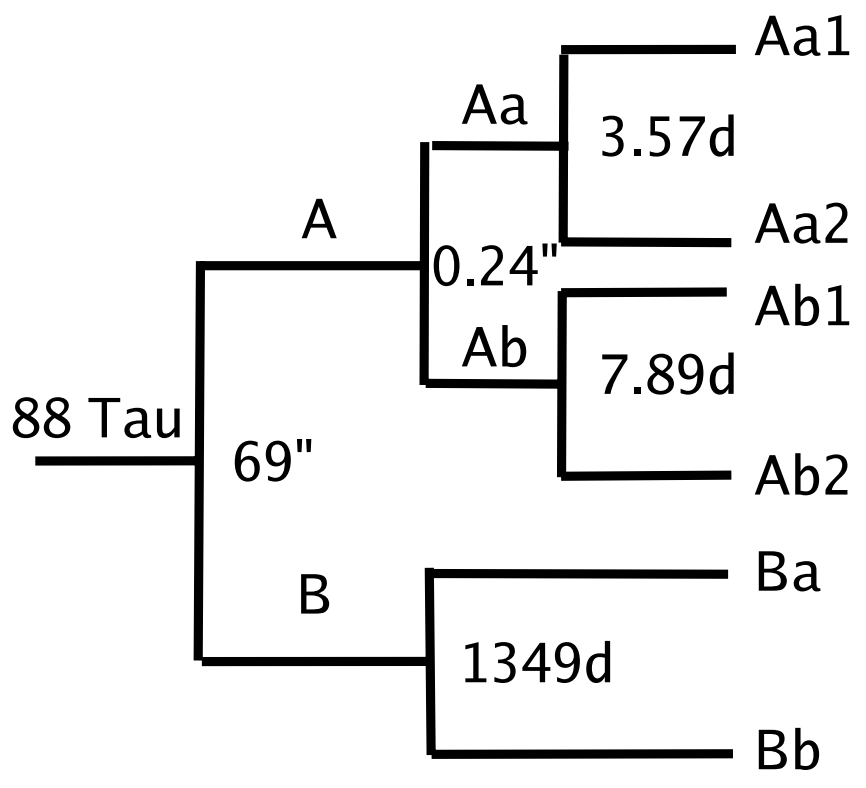

FIG. 1.-Schematic diagram of the 88 Tau system.

The obtained differential astrometry is listed in Table 1. Note that the astrometry on any single night is essentially that of a single-baseline interferometer, yielding a very small error in the direction aligned with the baseline, but limited to the effect of Earth-rotation synthesis in the perpendicular direction. The median minor-axis formal uncertainty is $10 \mu$ as, while the median majoraxis uncertainty is $312 \mu$ as. To properly weight the data set when doing a combined fit with previous astrometry and radial velocity data, we fit an orbital model to the PHASES astrometry by itself, and rescaled the formal uncertainties so as to yield a reduced $\chi^{2}$ of unity; the resulting scale factor was 2.5 , indicating a substantial amount of excess scatter beyond the internal error estimates. We do not believe this scatter to be due to the effect of starspots, given that the Hipparcos photometry of this system indicates a scatter of no more than 5 mmag; the resulting maximum starspot-induced astrometric noise would be $\sim 4 \mu$ as (Muterspaugh et al. 2006a). We have however identified possible instrumental sources of this systematic error and developed methods for reducing it; see M. W. Muterspaugh et al. (2007, in preparation). Nevertheless, the existing astrometry is sufficient to detect astrometric motion induced by the short-period subsystems.

\subsection{Previous Astrometry}

In addition to our astrometry, 88 Tau $\mathrm{A}$ has been followed by a number of observers with speckle-interferometric techniques. We use 20 observations tabulated in the Fourth Catalog of Interferometric Measurements of Binary Stars ${ }^{8}$ (Hartkopf et al. 2001) to further constrain our fit. Although of somewhat lower precision, the considerable time baseline (including observations dating from 1985) helps constrain the parameters of the wide orbit. In many cases the published astrometry lacks uncertainties, and we

${ }^{8}$ Available at http://ad.usno.navy.mil/wds/int4.html.

TABLE 1

PHASES Astrometric Data for 88 Tauri A

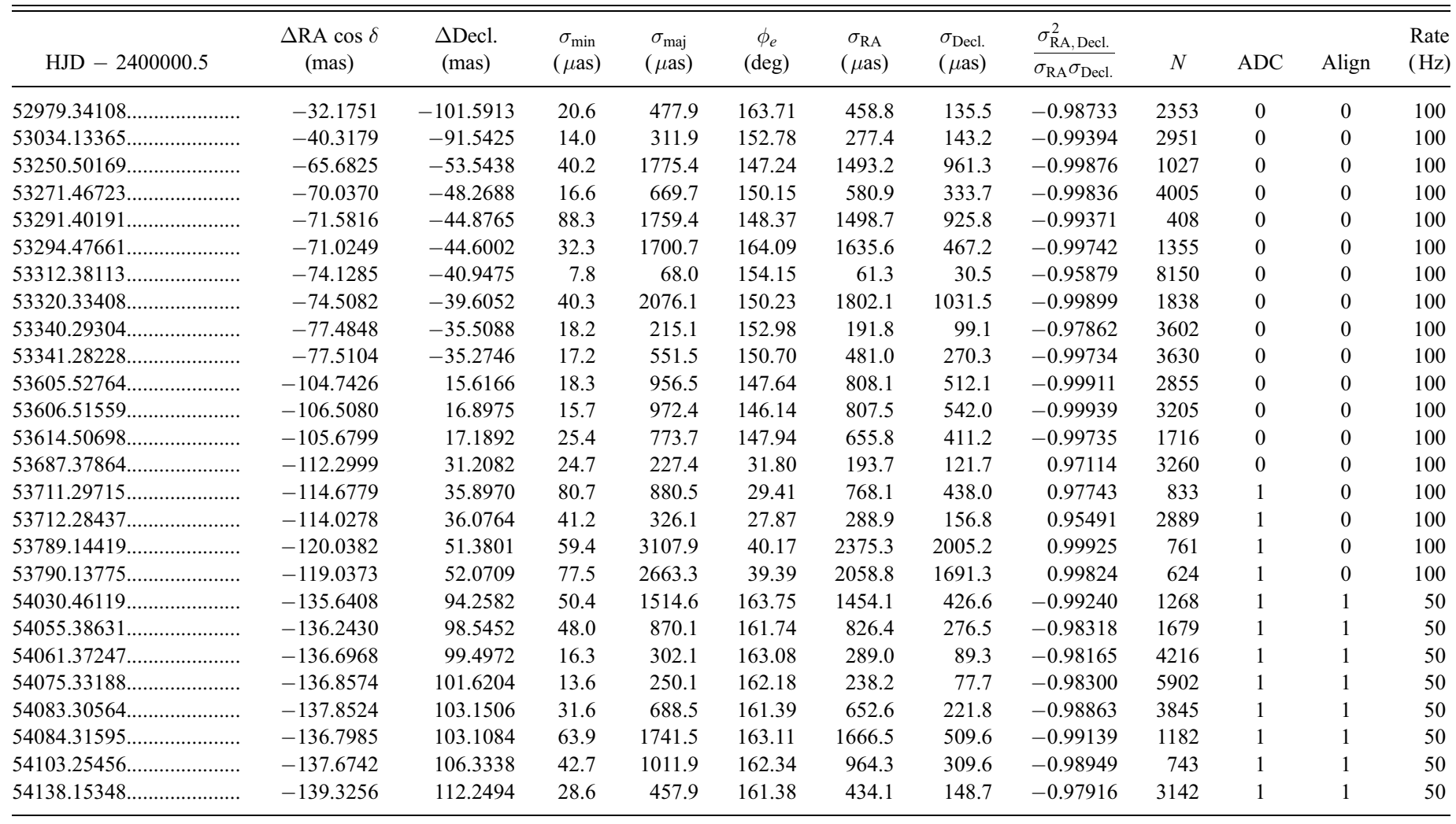

Notes.-All quantities are in the ICRS 2000.0 reference frame. The uncertainty values presented in these data have been scaled by a factor of 2.5 over the formal (internal) uncertainties for each night. The sixth column $\left(\phi_{e}\right)$ is the angle between the major axis of the uncertainty ellipse and the right ascension axis, measured from increasing differential right ascension through increasing differential declination. $N$ is the number of scans obtained in a night; each scan typically represents $0.5-1 \mathrm{~s}$ of integration. ADC indicates that the observations made use of the automatic dispersion compensator. "Rate" indicates the tracking rate of the fringe tracker used to stabilize the fringe phase during measurement. "Align" indicates whether or not the automatic alignment system was used to stabilize the system pupil. 
TABLE 2

KPNO Radial Velocity Data for 88 Tauri A

\begin{tabular}{|c|c|c|c|c|c|c|c|c|}
\hline HJD -2400000.5 & $\begin{array}{c}V_{\text {Aal }} \\
\left(\mathrm{km} \mathrm{s}^{-1}\right)\end{array}$ & Weight $^{\mathrm{a}}$ & $\begin{array}{c}V_{\mathrm{Aa} 2} \\
\left(\mathrm{~km} \mathrm{~s}^{-1}\right)\end{array}$ & Weight ${ }^{\mathrm{b}}$ & $\begin{array}{c}V_{\mathrm{Ab} 1} \\
\left(\mathrm{~km} \mathrm{~s}^{-1}\right)\end{array}$ & Weight $^{\mathrm{c}}$ & $\begin{array}{c}V_{\mathrm{Ab} 2} \\
\left(\mathrm{~km} \mathrm{~s}^{-1}\right)\end{array}$ & Weight $^{\mathrm{d}}$ \\
\hline 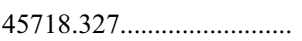 & 91.4 & 1.0 & -66.5 & 1.0 & .. & $\ldots$ & $\ldots$ & 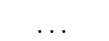 \\
\hline 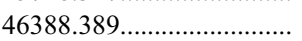 & -48.7 & 1.0 & 141.6 & 1.0 & 40.1 & 1.0 & 1.1 & 1.0 \\
\hline 46390.212 & 99.8 & 1.0 & -85.2 & 1.0 & -5.8 & 1.0 & $\ldots$ & $\ldots$ \\
\hline 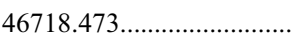 & 96.5 & 1.0 & -93.7 & 1.0 & 59.8 & 0.4 & -4.5 & 1.0 \\
\hline 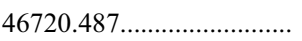 & -51.6 & 1.0 & 139.5 & 1.0 & $\ldots$ & $\ldots$ & $\ldots$ & $\ldots$ \\
\hline 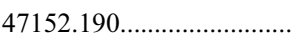 & -45.3 & 1.0 & 123.6 & 1.0 & 57.9 & 1.0 & $\ldots$ & $\ldots$ \\
\hline 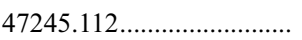 & -50.2 & 1.0 & 131.5 & 1.0 & $\ldots$ & $\ldots$ & $\ldots$ & $\ldots$ \\
\hline $47456.264 \ldots \ldots \ldots \ldots \ldots \ldots \ldots$ & -52.2 & 1.0 & 129.7 & 1.0 & 9.3 & 1.0 & $\ldots$ & $\ldots$ \\
\hline 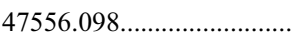 & -57.7 & 1.0 & 135.8 & 1.0 & $\ldots$ & $\ldots$ & $\ldots$ & $\ldots$ \\
\hline 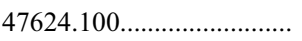 & -53.5 & 1.0 & 125.7 & 1.0 & $\ldots$ & $\ldots$ & $\ldots$ & $\ldots$ \\
\hline 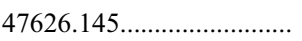 & 66.5 & 1.0 & -57.7 & 1.0 & $\ldots$ & $\ldots$ & 4.3 & 1.0 \\
\hline 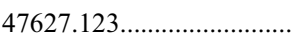 & -49.3 & 1.0 & 118.9 & 1.0 & $\ldots$ & $\ldots$ & $\ldots$ & $\ldots$ \\
\hline 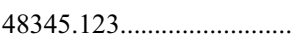 & -60.5 & 1.0 & 133.2 & 1.0 & $\ldots$ & $\ldots$ & $\ldots$ & $\ldots$ \\
\hline 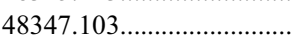 & 96.5 & 1.0 & -102.4 & 1.0 & 9.4 & 1.0 & 69.9 & 0.4 \\
\hline 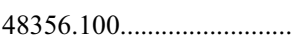 & -58.6 & 1.0 & 128.6 & 1.0 & 10.0 & 1.0 & 59.1 & 1.0 \\
\hline 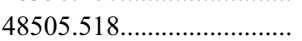 & -46.5 & 1.0 & 106.1 & 1.0 & 14.0 & 1.0 & 53.0 & 1.0 \\
\hline $48573.407 \ldots \ldots \ldots \ldots \ldots \ldots$ & -49.1 & 1.0 & 112.7 & 1.0 & 42.5 & 0.4 & 26.2 & 0.4 \\
\hline 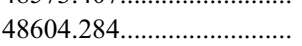 & 90.2 & 1.0 & -98.1 & 1.0 & 57.7 & 0.4 & 15.4 & 1.0 \\
\hline 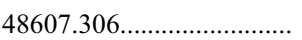 & 75.8 & 1.0 & -74.8 & 1.0 & 3.0 & 1.0 & $\ldots$ & $\ldots$ \\
\hline 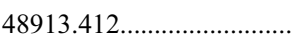 & -43.0 & 1.0 & 105.6 & 1.0 & 19.0 & 1.0 & 50.9 & 1.0 \\
\hline $48916.375 \ldots \ldots \ldots \ldots \ldots \ldots \ldots \ldots$ & -55.5 & 1.0 & 125.2 & 1.0 & $\ldots$ & $\ldots$ & $\ldots$ & $\ldots$ \\
\hline 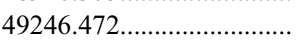 & 76.6 & 1.0 & -67.1 & 1.0 & 6.6 & 0.4 & $\ldots$ & $\ldots$ \\
\hline 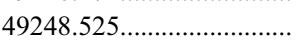 & -56.1 & 1.0 & 131.7 & 1.0 & 44.0 & 1.0 & 15.3 & 1.0 \\
\hline $49250.537 \ldots \ldots \ldots \ldots \ldots \ldots \ldots \ldots$ & 101.6 & 1.0 & -93.6 & 1.0 & 58.9 & 0.4 & 1.7 & 1.0 \\
\hline 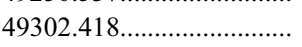 & -53.7 & 1.0 & 132.4 & 1.0 & 12.9 & 1.0 & 51.9 & 1.0 \\
\hline 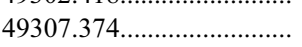 & 91.3 & 1.0 & -87.2 & 1.0 & 23.0 & 0.4 & 34.6 & 0.4 \\
\hline 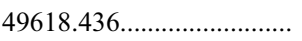 & 94.0 & 1.0 & -92.1 & 1.0 & $\ldots$ & $\ldots$ & $\ldots$ & $\ldots$ \\
\hline 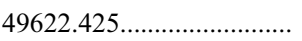 & 60.6 & 1.0 & -41.1 & 1.0 & $\ldots$ & $\ldots$ & $\ldots$ & $\ldots$ \\
\hline 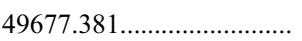 & -50.0 & 1.0 & 132.6 & 1.0 & $\ldots$ & $\ldots$ & $\ldots$ & $\ldots$ \\
\hline 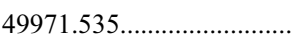 & 93.8 & 1.0 & -78.7 & 1.0 & -9.8 & 1.0 & 53.5 & 0.4 \\
\hline 49973.434................................ & -48.2 & 1.0 & 138.8 & 1.0 & $\ldots$ & $\ldots$ & $\ldots$ & $\ldots$ \\
\hline 49973.535 & -51.1 & 1.0 & 142.9 & 1.0 & 17.0 & 0.4 & 26.0 & 0.4 \\
\hline 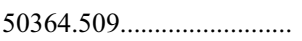 & 102.5 & 1.0 & -85.3 & 1.0 & 5.8 & 0.4 & 30.0 & 0.4 \\
\hline 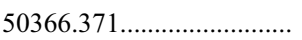 & -48.5 & 1.0 & 146.0 & 1.0 & $\ldots$ & $\ldots$ & $\ldots$ & $\ldots$ \\
\hline $50400.392 \ldots \ldots \ldots \ldots \ldots \ldots \ldots$ & 100.7 & 1.0 & -88.4 & 1.0 & 38.2 & 1.0 & -1.3 & 1.0 \\
\hline 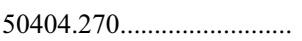 & 91.1 & 1.0 & -66.5 & 1.0 & $\ldots$ & $\ldots$ & $\ldots$ & $\ldots$ \\
\hline 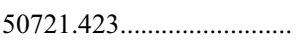 & 93.8 & 1.0 & -67.1 & 1.0 & -9.9 & 1.0 & 38.0 & 0.4 \\
\hline 50721.501.............................. & 96.1 & 1.0 & -77.1 & 1.0 & -11.3 & 1.0 & 40.2 & 1.0 \\
\hline 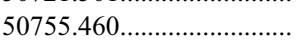 & -46.1 & 1.0 & 139.7 & 1.0 & 38.8 & 1.0 & -11.3 & 0.4 \\
\hline $50757.384 \ldots \ldots$ & 104.0 & 1.0 & -86.7 & 1.0 & 34.0 & 1.0 & -2.7 & 1.0 \\
\hline $50832.293 \ldots \ldots \ldots \ldots \ldots \ldots$ & 104.7 & 1.0 & -82.2 & 1.0 & -9.7 & 1.0 & 44.2 & 1.0 \\
\hline 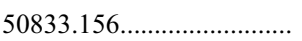 & 57.7 & 1.0 & $\ldots$ & $\ldots$ & $\ldots$ & $\ldots$ & $\ldots$ & $\ldots$ \\
\hline 51088.384................................. & -7.6 & 1.0 & 92.2 & 1.0 & $\ldots$ & $\ldots$ & $\ldots$ & $\ldots$ \\
\hline 51089.481.................................. & 106.0 & 1.0 & -85.2 & 1.0 & $\ldots$ & $\ldots$ & $\ldots$ & $\ldots$ \\
\hline 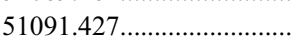 & -49.1 & 1.0 & 149.9 & 1.0 & -18.8 & 0.4 & 47.3 & 1.0 \\
\hline 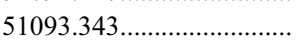 & 103.8 & 1.0 & -79.4 & 1.0 & 3.6 & 0.4 & 22.0 & 0.4 \\
\hline 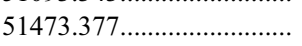 & -51.8 & 1.0 & 147.2 & 1.0 & 43.5 & 1.0 & -18.3 & 0.4 \\
\hline $51475.281 \ldots \ldots \ldots \ldots \ldots \ldots \ldots \ldots \ldots$ & 107.5 & 1.0 & -85.7 & 1.0 & $\ldots$ & $\ldots$ & $\ldots$ & $\ldots$ \\
\hline 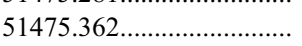 & 107.8 & 1.0 & -85.7 & 1.0 & 22.1 & 0.4 & 4.1 & 0.4 \\
\hline $51803.489 \ldots \ldots \ldots \ldots \ldots \ldots \ldots \ldots$ & 95.8 & 1.0 & -66.7 & 1.0 & $\ldots$ & $\ldots$ & $\ldots$ & $\ldots$ \\
\hline 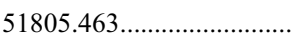 & -49.1 & 1.0 & 147.2 & 1.0 & 41.6 & 1.0 & -21.2 & 0.4 \\
\hline 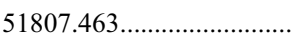 & 107.0 & 1.0 & -86.9 & 1.0 & 5.1 & 0.4 & 22.7 & 0.4 \\
\hline 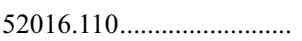 & -46.8 & 1.0 & 142.6 & 1.0 & $\ldots$ & $\ldots$ & $\ldots$ & $\ldots$ \\
\hline 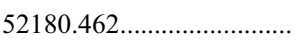 & -49.0 & 1.0 & 146.2 & 1.0 & -14.7 & 1.0 & 43.8 & 1.0 \\
\hline 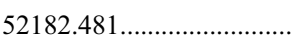 & 104.0 & 1.0 & -86.2 & 1.0 & 28.2 & 0.4 & 0.4 & 0.4 \\
\hline 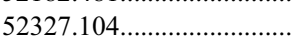 & -48.8 & 1.0 & 145.5 & 1.0 & 35.9 & 1.0 & -0.9 & 0.4 \\
\hline 52329.148................................ & 89.8 & 1.0 & -64.0 & 1.0 & -8.0 & 0.4 & 38.2 & 0.4 \\
\hline 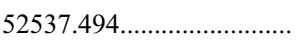 & -50.0 & 1.0 & 137.7 & 1.0 & 35.0 & 1.0 & -4.0 & 0.4 \\
\hline $52539.468 \ldots \ldots \ldots \ldots \ldots \ldots \ldots \ldots \ldots$ & 106.0 & 1.0 & -89.1 & 1.0 & 42.9 & 1.0 & -7.0 & 1.0 \\
\hline 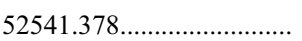 & -46.3 & 1.0 & 144.8 & 1.0 & $\ldots$ & $\ldots$ & $\ldots$ & $\ldots$ \\
\hline $52541.473 \ldots \ldots \ldots \ldots \ldots \ldots \ldots \ldots \ldots$ & -45.5 & 1.0 & 139.3 & 1.0 & 1.5 & 0.4 & 38.9 & 1.0 \\
\hline 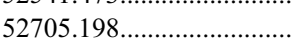 & -45.8 & 1.0 & 126.6 & 1.0 & 48.5 & 1.0 & $\ldots$ & $\ldots$ \\
\hline $52707.190 \ldots \ldots \ldots \ldots \ldots \ldots$ & 101.6 & 1.0 & -88.8 & 1.0 & $\ldots$ & $\ldots$ & $\cdots$ & $\cdots$ \\
\hline 52709.127 & -54.6 & 1.0 & 144.6 & 1.0 & $\cdots$ & $\begin{array}{l}\cdots \\
\ldots\end{array}$ & 31.1 & 1.0 \\
\hline
\end{tabular}


TABLE 2-Continued

\begin{tabular}{|c|c|c|c|c|c|c|c|c|}
\hline HJD -2400000.5 & $\begin{array}{c}V_{\mathrm{Aa} 1} \\
\left(\mathrm{~km} \mathrm{~s}^{-1}\right)\end{array}$ & Weight $^{\mathrm{a}}$ & $\begin{array}{c}V_{\mathrm{Aa} 2} \\
\left(\mathrm{~km} \mathrm{~s}^{-1}\right)\end{array}$ & Weight $^{\mathrm{b}}$ & $\begin{array}{c}V_{\mathrm{Ab} 1} \\
\left(\mathrm{~km} \mathrm{~s}^{-1}\right)\end{array}$ & Weight $^{\mathrm{c}}$ & $\begin{array}{c}V_{\mathrm{Ab} 2} \\
\left(\mathrm{~km} \mathrm{~s}^{-1}\right)\end{array}$ & Weight $^{\mathrm{d}}$ \\
\hline 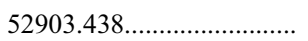 & 93.6 & 1.0 & -75.7 & 1.0 & $\ldots$ & $\ldots$ & $\ldots$ & . \\
\hline $52941.345 \ldots \ldots \ldots \ldots \ldots \ldots \ldots$ & -50.8 & 1.0 & 141.1 & 1.0 & 51.1 & 1.0 & -6.8 & 0.4 \\
\hline $53273.479 \ldots \ldots \ldots \ldots \ldots \ldots \ldots$ & -53.1 & 1.0 & 139.3 & 1.0 & 41.0 & 1.0 & 7.9 & 1.0 \\
\hline $53278.490 \ldots \ldots \ldots \ldots \ldots \ldots \ldots \ldots$ & 93.6 & 1.0 & -84.9 & 1.0 & $\ldots$ & $\ldots$ & $\ldots$ & $\ldots$ \\
\hline 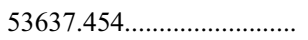 & -55.3 & 1.0 & 134.5 & 1.0 & 12.9 & 1.0 & 42.4 & 1.0 \\
\hline 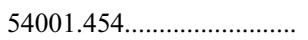 & -42.5 & 1.0 & 113.7 & 1.0 & 3.6 & 0.4 & 66.6 & 1.0 \\
\hline $54003.423 \ldots \ldots \ldots \ldots \ldots \ldots \ldots \ldots$ & 89.9 & 1.0 & -90.3 & 1.0 & $\ldots$ & .. & .. & $\ldots$ \\
\hline 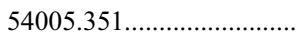 & -58.5 & 1.0 & 137.5 & 1.0 & 62.1 & 1.0 & $\ldots$ & $\ldots$ \\
\hline $54005.506 \ldots \ldots \ldots \ldots \ldots$ & -57.7 & 1.0 & 134.6 & 1.0 & 60.4 & 1.0 & 0.9 & 0.4 \\
\hline
\end{tabular}

${ }^{\text {a }}$ An observation of Aal of unit weight has a standard error of $2.0 \mathrm{~km} \mathrm{~s}^{-1}$.

${ }^{b}$ An observation of Aa2 of unit weight has a standard error of $2.3 \mathrm{~km} \mathrm{~s}^{-1}$.

c An observation of Ab1 of unit weight has a standard error of $2.6 \mathrm{~km} \mathrm{~s}^{-1}$.

${ }^{d}$ An observation of Ab2 of unit weight has a standard error of $2.6 \mathrm{~km} \mathrm{~s}^{-1}$.

therefore assigned a plausible initial uncertainty of 3 mas in separation and $2^{\circ}$ in position angle to these points (we used published uncertainties for the points where such were available). We then performed a least-squares fit of a single Keplerian orbital model (corresponding to the Aa-Ab orbit; the subsystems are far too small to be detected by these data), and scaled all of the uncertainties so as to yield a reduced $\chi^{2}$ of unity. We find the average uncertainty in separation to be 5 mas, and the average position-angle uncertainty to be $3^{\circ}$.

\subsection{Spectroscopic Observations and Reductions}

From 1984 January through 2006 September we obtained 82 spectrograms of 88 Tau with the Kitt Peak National Observatory (KPNO) 0.9 m coudé feed telescope, coudé spectrograph, and a TI CCD detector. Sixty-eight spectrograms are centered in the red at $6430 \AA$, cover a wavelength range of about $80 \AA$, and have a 2 pixel resolution of $0.21 \AA$. Those spectra have signal-tonoise ratios of $\sim 250$. The remaining 14 spectrograms are centered in the blue at $4500 \AA$, cover a wavelength range of $85 \AA$, and have a resolution of $0.22 \AA$. Signal-to-noise ratios of $\sim 300$ are typical.

From 2004 January through 2007 April we acquired 29 spectrograms with the Tennessee State University $2 \mathrm{~m}$ automatic spectroscopic telescope (AST), fiber-fed echelle spectrograph, and a $2048 \times 4096$ SITe ST-002A CCD. The echelle spectrograms have 21 orders, covering the wavelength range $4920-7100 \AA$ with an average resolution of $0.17 \AA$. The typical signal-to-noise ratio is $\sim 50$. Eaton \& Williamson (2004) have given a more extensive description of the telescope, situated at Fairborn Observatory near Washington Camp in the Patagonia Mountains of southeastern Arizona, and its operation.

For the KPNO spectrograms we determined radial velocities with the IRAF cross-correlation program FXCOR (Fitzpatrick 1993), fitting Gaussian functions to the individual cross-correlation peaks. Double-Gaussian fits were required to obtain individual velocities from blended cross-correlation peaks. The IAU radial velocity standard star 10 Tau was used as the cross-correlation reference star for the red-wavelength spectrograms. Its velocity of $27.9 \mathrm{~km} \mathrm{~s}^{-1}$ was adopted from Scarfe et al. (1990). Lines in the wavelength region redward of $6445 \AA$ are not particularly suitable for measurement because most features are blends, and there are a number of modest-strength water vapor lines. Thus, the radial velocities were determined from lines in the region 6385 $6445 \AA$. However, this $60 \AA$ portion of the spectrum is so small that a spectrum mismatch, caused by the varying strength of line blends with temperature, between the $\mathrm{A}$ and $\mathrm{F}$ spectral type components of 88 Tau and the F9 IV-V (Keenan \& McNeil 1989) spectral type of the reference star $10 \mathrm{Tau}$, can significantly alter the measured velocity. Thus, instead of cross-correlating this entire $60 \AA$ wavelength region, only the wavelength regions around two or three of the strongest and least-blended lines, usually the $\mathrm{Fe}$ I lines at 6394 and $6412 \AA$ plus the Ca I line at $6439 \AA$, were crosscorrelated.

At blue wavelengths the Am star dominates the spectrum. To compute velocities from those spectrograms, 68 Tau, spectral type A2 IV (Abt \& Morrell 1995), which has a velocity of $39.0 \mathrm{~km} \mathrm{~s}^{-1}$ (Fekel 1999), was used as the reference star. The region between 4485 and $4525 \AA$ was cross correlated. Velocities for our KPNO spectra are given in Table 2.

For the Fairborn Observatory AST spectra, lines in approximately 100 regions, centered on the rest wavelengths (Moore et al. 1966) of relatively strong lines (mostly of $\mathrm{Fe}_{\mathrm{I}}$ and $\mathrm{Fe}$ II) that were not strong blends, were measured. Lines at the ends of each echelle order were excluded because of their lower signal-to-noise ratios. A Gaussian function was fitted to the profile of each component. Double-Gaussian fits were required to represent blended components. The difference between the observed wavelength and that given in the solar line list of Moore et al. (1966) was used to compute the radial velocity, and a heliocentric correction was applied. The final mean velocity for each observation is given in Table 3. Unpublished velocities of several IAU standard stars with F dwarf spectral types indicate that the Fairborn Observatory velocities have a small zero-point offset of $-0.3 \mathrm{~km} \mathrm{~s}^{-1}$ relative to the velocities of Scarfe et al. (1990).

\subsection{Preliminary Spectroscopic Analysis}

In a study of lithium in Am stars Burkhart \& Coupry (1988) acquired two high-resolution spectrograms of 88 Tau in the $6710 \AA$ A region. Comparing two sets of lines in the two spectra, they reported detecting the lines of five different components. Figure 2 presents two spectra of 88 Tau A in the 6430 Å region that show the two components of the 3.57 day binary near opposite nodes in their spectroscopic orbit, when the components have their maximum velocity separation. Between the two "outside" lines are two additional weak components. From a careful inspection of our KPNO spectra, as well as the ones obtained at Fairborn Observatory, we find lines of only four components, rather than the five reported by Burkhart \& Coupry (1988). In Figure 2 many lines of the Am star are $\sim 5 \%$ deep, while the lines of the other three components typically have line depths $\leq 2.5 \%$. Correctly identifying components in such a weak-lined and complex spectrum is not easy because in many spectra two or more of the components are blended. 
TABLE 3

Fairborn Observatory Radial Velocity Data for 88 Tauri A

\begin{tabular}{|c|c|c|c|c|c|c|c|c|}
\hline HJD - 2400000.5 & $\begin{array}{c}V_{\mathrm{Aal} 1} \\
\left(\mathrm{~km} \mathrm{~s}^{-1}\right)\end{array}$ & Weight ${ }^{\mathrm{a}}$ & $\begin{array}{c}V_{\mathrm{Aa} 2} \\
\left(\mathrm{~km} \mathrm{~s}^{-1}\right)\end{array}$ & Weight ${ }^{b}$ & $\begin{array}{c}V_{\mathrm{Ab} 1} \\
\left(\mathrm{~km} \mathrm{~s}^{-1}\right)\end{array}$ & Weight $^{\mathrm{c}}$ & $\begin{array}{c}V_{\mathrm{Ab} 2} \\
\left(\mathrm{~km} \mathrm{~s}^{-1}\right)\end{array}$ & Weight $^{\mathrm{d}}$ \\
\hline $53020.204 \ldots \ldots \ldots \ldots \ldots \ldots \ldots$ & -38.5 & 1.0 & 118.0 & 1.0 & 50.8 & 1.0 & $\ldots$ & $\ldots$ \\
\hline $53032.197 \ldots \ldots \ldots \ldots \ldots \ldots \ldots$ & 101.1 & 1.0 & -90.3 & 1.0 & -6.3 & 1.0 & 53.7 & 1.0 \\
\hline $53052.273 \ldots \ldots \ldots \ldots \ldots \ldots \ldots \ldots \ldots$ & -43.0 & 1.0 & 126.0 & 1.0 & 50.9 & 1.0 & $\ldots$ & $\ldots$ \\
\hline $53276.489 \ldots \ldots \ldots \ldots \ldots \ldots \ldots$ & -37.3 & 1.0 & 110.0 & 1.0 & $\ldots$ & 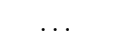 & 53.0 & 1.0 \\
\hline $53285.468 \ldots \ldots \ldots \ldots \ldots \ldots \ldots \ldots \ldots$ & 85.9 & 1.0 & -69.9 & 1.0 & 10.8 & 1.0 & 41.8 & 1.0 \\
\hline $53314.392 \ldots \ldots \ldots \ldots \ldots \ldots \ldots \ldots$ & 102.0 & 1.0 & -95.0 & 1.0 & 8.8 & 1.0 & 40.6 & 1.0 \\
\hline $53350.390 \ldots \ldots \ldots \ldots \ldots \ldots \ldots \ldots$ & 93.6 & 1.0 & -85.3 & 1.0 & 46.3 & 1.0 & 7.7 & 1.0 \\
\hline 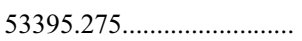 & -28.2 & 1.0 & 97.3 & 1.0 & -2.4 & 1.0 & 58.0 & 1.0 \\
\hline 53405.184.............................. & -44.4 & 1.0 & 127.0 & 1.0 & 39.9 & 1.0 & 11.9 & 1.0 \\
\hline $53630.518 \ldots \ldots \ldots \ldots \ldots \ldots \ldots \ldots \ldots$ & -55.6 & 1.0 & 139.5 & 1.0 & 2.7 & 1.0 & 57.9 & 1.0 \\
\hline $53644.477 \ldots \ldots \ldots \ldots \ldots \ldots \ldots$ & -48.0 & 1.0 & 127.2 & 1.0 & 38.3 & 1.0 & 20.1 & 1.0 \\
\hline $53659.486 \ldots \ldots \ldots \ldots \ldots \ldots \ldots \ldots$ & -33.5 & 1.0 & 102.9 & 1.0 & 58.4 & 1.0 & $\ldots$ & $\ldots$ \\
\hline $53700.387 \ldots \ldots \ldots \ldots \ldots \ldots \ldots$ & 90.5 & 1.0 & -82.3 & 1.0 & $\ldots$ & $\ldots$ & $\ldots$ & $\ldots$ \\
\hline 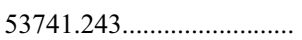 & -56.5 & 1.0 & 139.1 & 1.0 & -1.2 & 1.0 & 56.7 & 1.0 \\
\hline 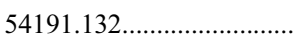 & -61.0 & 1.0 & 136.8 & 1.0 & 7.3 & 1.0 & 63.7 & 1.0 \\
\hline $54194.134 \ldots \ldots \ldots \ldots \ldots \ldots \ldots \ldots$ & -28.7 & 1.0 & 85.1 & 1.0 & $\ldots$ & $\ldots$ & $\ldots$ & $\ldots$ \\
\hline $54194.156 \ldots \ldots \ldots \ldots \ldots \ldots \ldots \ldots \ldots$ & -30.2 & 1.0 & 89.5 & 1.0 & $\ldots$ & $\ldots$ & $\ldots$ & $\ldots$ \\
\hline 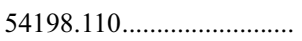 & -56.9 & 1.0 & 132.4 & 1.0 & $\ldots$ & $\ldots$ & $\ldots$ & $\ldots$ \\
\hline
\end{tabular}

${ }^{\mathrm{a}}$ An observation of Aa1 of unit weight has a standard error of $2.0 \mathrm{~km} \mathrm{~s}^{-1}$.

${ }^{b}$ An observation of Aa2 of unit weight has a standard error of $2.3 \mathrm{~km} \mathrm{~s}^{-1}$.

c An observation of Ab1 of unit weight has a standard error of $2.6 \mathrm{~km} \mathrm{~s}^{-1}$.

${ }^{\mathrm{d}}$ An observation of $\mathrm{Ab} 2$ of unit weight has a standard error of $2.6 \mathrm{~km} \mathrm{~s}^{-1}$.

While the identification of the components of the 3.57 day binary is straightforward, the very weak lines of the other two components are similar in strength and line width, making it difficult to tell them apart. To determine a preliminary orbital period, we initially examined only the latter portion of our KPNO velocities, obtained from MJD 50,400 to 54,000. For each observation we computed the absolute value of the velocity difference between the two components and then used those results as the input data for two different period-finding approaches. First, a sine curve was fitted to the velocity differences for trial periods between 1 and 100 days with a step size of 0.0005 days. The period with the smallest sum of the squared residuals was adopted as the best period.

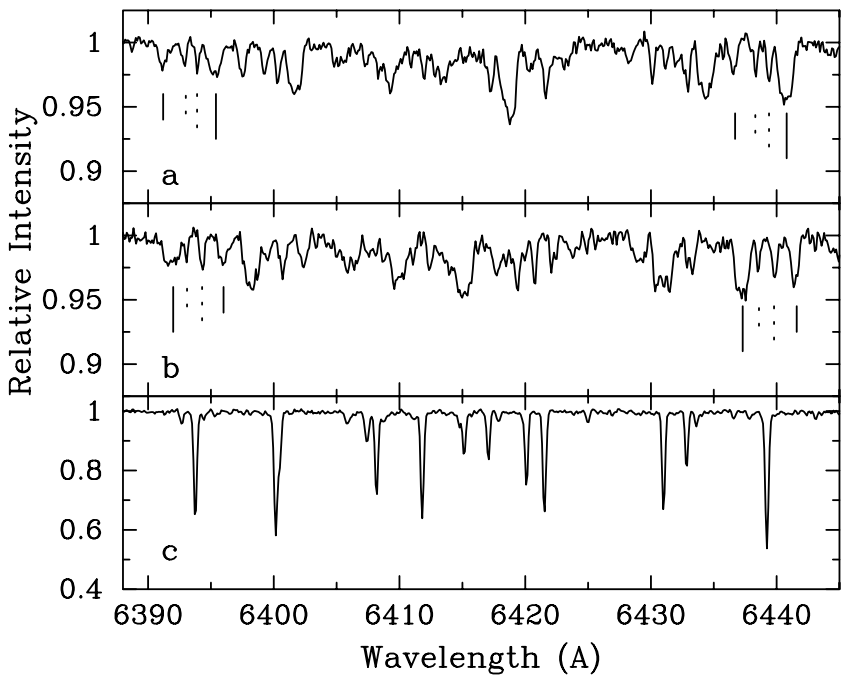

FIG. 2.-Two spectra of 88 Tau, (a) JD 2,452,539.97 and (b) JD 2,454,006.01, compared with $(c)$ the spectrum of the IAU radial velocity standard 10 Tau. The four components of 88 Tau A are identified for two different lines. Solid tick marks indicate lines of the $\mathrm{Aa}$ ( 3.57 day period) binary, with the longer tick mark identifying the primary. Dotted tick marks indicate lines of the $\mathrm{Ab}$ ( 7.89 day period) binary, with the longer tick mark identifying the primary.
Next, a search over a similar period range and with the same step size was done with the least-string method (Bopp et al. 1970). Both searches resulted in a period of 3.9435 days. Doubling this period produced an orbital period of 7.887 days. Separate analyses of our earlier KPNO velocities as well as the Fairborn Observatory velocities produced a similar orbital period. We then adopted the 7.887 day period and computed a phase diagram to identify correctly the components. Afterward we compared those results with an attempt at visual identification, based on which set of lines appeared to be stronger in each spectrum. The visual inspection correctly identified the more massive component only about half of the time. Apparently, the lines of these two components are similar enough that weak lines from other components and noise can significantly affect the apparent line strengths. Thus, we conclude that in our spectra it is not possible to differentiate the two components based on line strength.

\subsection{Orbital Models}

In modeling the hierarchical quadruple system we make the simplifying assumption that the three orbital systems do not perturb each other during the time of our observations; i.e., we use three Keplerian orbital systems, one wide (Aa-Ab) and slow (18 yr period), and two short-period systems: Aa1-Aa2, 3.57 day period, and $\mathrm{Ab} 1-\mathrm{Ab} 2,7.89$ day period. Note that one cannot simply superimpose the separation vectors from the three models; this is because the PHASES observable is the angle between the two centers of light (COL) of the short-period systems:

$$
\begin{aligned}
\boldsymbol{y}_{\mathrm{obs}}= & \boldsymbol{r}_{\mathrm{Aa}-\mathrm{Ab}}+\frac{R_{\mathrm{Aa}}-L_{\mathrm{Aa}}}{\left(1+R_{\mathrm{Aa}}\right)\left(1+L_{\mathrm{Aa}}\right)} \boldsymbol{r}_{\mathrm{Aa} 1-\mathrm{Aa} 2} \\
& -\frac{R_{\mathrm{Ab}}-L_{\mathrm{Ab}}}{\left(1+R_{\mathrm{Ab}}\right)\left(1+L_{\mathrm{Ab}}\right)} \boldsymbol{r}_{\mathrm{Ab} 1-\mathrm{Ab} 2 .} .
\end{aligned}
$$

Here $R_{\mathrm{Aa}}=M_{\mathrm{Aa} 2} / M_{\mathrm{Aa} 1}$ is the Aa component mass ratio and $L_{\mathrm{Aa}}=$ $L_{\mathrm{Aa} 2} / L_{\mathrm{Aa} 1}$ the luminosity ratio, while $R_{\mathrm{Ab}}=M_{\mathrm{Ab} 2} / M_{\mathrm{Ab} 1}$ and $L_{\mathrm{Ab}}=$ $L_{\mathrm{Ab} 2} / L_{\mathrm{Ab} 1}$ are the corresponding ratios for the $\mathrm{Ba}-\mathrm{Bb}$ subsystem. 
TABLE 4

Best-Fit Orbital Parameters for 88 Tauri A

\begin{tabular}{|c|c|c|}
\hline Parameter & Value and Uncertainty & Previous Value \\
\hline$\chi^{2}$. & 547 & $\ldots$ \\
\hline 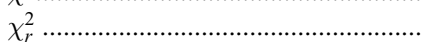 & 1.37 & $\ldots$ \\
\hline Number of parameters ......................... & 23 & $\ldots$ \\
\hline \multicolumn{3}{|l|}{$\mathrm{Aa}-\mathrm{Ab}:$} \\
\hline 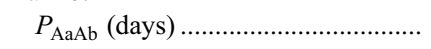 & $6585 \pm 12$ & $6593 \pm 44^{\mathrm{a}}$ \\
\hline 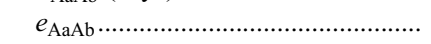 & $0.0715 \pm 0.0026$ & $0.083 \pm 0.008^{\mathrm{a}}$ \\
\hline 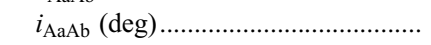 & $69.923 \pm 0.048$ & $70.4 \pm 0.4^{\mathrm{a}}$ \\
\hline 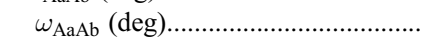 & $205.7 \pm 1.2$ & $222.0 \pm 3.0^{\mathrm{a}}$ \\
\hline 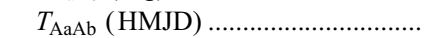 & $55261 \pm 22$ & $\ldots$ \\
\hline 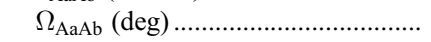 & $146.734 \pm 0.067$ & $146.6 \pm 0.3^{\mathrm{a}}$ \\
\hline 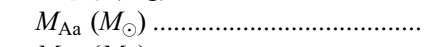 & $3.42 \pm 0.18$ & $\ldots$ \\
\hline 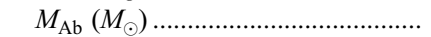 & $2.13 \pm 0.13$ & $\ldots$ \\
\hline 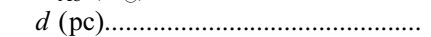 & $50.70 \pm 0.88$ & $\ldots$ \\
\hline \multicolumn{3}{|l|}{ Aa1-Aa2: } \\
\hline$P_{\mathrm{Aa} 1 \mathrm{Aa} 2}($ days $) \ldots \ldots \ldots \ldots \ldots$ & $3.571096 \pm 0.000003$ & $3.571391 \pm 0.000008^{b}$ \\
\hline 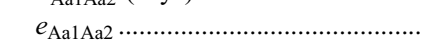 & 0.0 & $0^{\mathrm{b}}$ \\
\hline 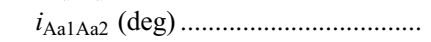 & $110.6 \pm 2.7$ & $\ldots$ \\
\hline 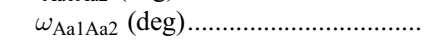 & 0.0 & $\ldots$ \\
\hline 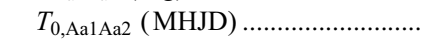 & $53389.3824 \pm 0.0030$ & $43108.103 \pm 0.013^{\mathrm{b}}$ \\
\hline 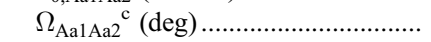 & $287.5 \pm 1.8$ & $\ldots$ \\
\hline 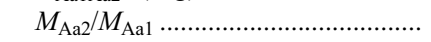 & $0.6602 \pm 0.0028$ & $\ldots$ \\
\hline$L_{\mathrm{Aa} 2} / L_{\mathrm{Aa} 1}(K \text { band })^{\mathrm{c}} \ldots \ldots \ldots \ldots \ldots \ldots \ldots$ & $0.249 \pm 0.035$ & $\ldots$ \\
\hline \multicolumn{3}{|l|}{$\mathrm{Ab} 1-\mathrm{Ab} 2:$} \\
\hline 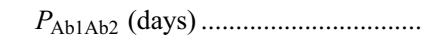 & $7.886969 \pm 0.000066$ & $\ldots$ \\
\hline 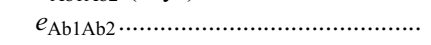 & 0.0 & $\ldots$ \\
\hline 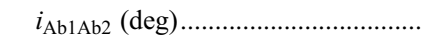 & $27.23 \pm 0.72$ & $\ldots$ \\
\hline$\omega_{\mathrm{Ab} 1 \mathrm{Ab} 2}(\mathrm{deg})$ & 0.0 & $\ldots$ \\
\hline 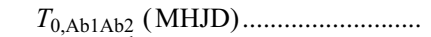 & $52507.31 \pm 0.02$ & $\ldots$ \\
\hline 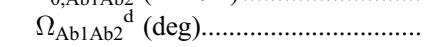 & $34.0 \pm 8.2$ & $\ldots$ \\
\hline 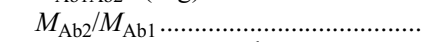 & $0.988 \pm 0.024$ & $\ldots$ \\
\hline$L_{\mathrm{Ab} 2} / L_{\mathrm{Ab} 1}(K \text { band })^{\mathrm{d}} \ldots \ldots \ldots \ldots \ldots \ldots \ldots \ldots \ldots \ldots \ldots \ldots$ & $0.865 \pm 0.028$ & $\ldots$ \\
\hline$V_{0}(\mathrm{KPNO})\left(\mathrm{km} \mathrm{s}^{-1}\right) \ldots \ldots \ldots \ldots \ldots \ldots$ & $23.70 \pm 0.17$ & $\ldots$ \\
\hline$V_{0}($ Fairborn $)\left(\mathrm{km} \mathrm{s}^{-1}\right) \ldots \ldots \ldots \ldots \ldots \ldots$ & $23.91 \pm 0.31$ & $\ldots$ \\
\hline
\end{tabular}

a From Balega et al. (1999).

b From Abt \& Levy (1985).

c An alternate, but disfavored, solution has $L_{\mathrm{Aa} 2} / L_{\mathrm{Aa} 1}=1.48$ and $\Omega_{\mathrm{Aa} 1 \mathrm{Aa} 2}=326$.

${ }^{\mathrm{d}}$ An alternate solution has $L_{\mathrm{Ab} 2} / L_{\mathrm{Ab} 1}=1.10$ and $\Omega_{\mathrm{Ab} 1 \mathrm{Ab} 2}=205$.

Including this coupling term for astrometric data is important when a full analysis, including radial velocity data, is made.

\section{RESULTS}

The best-fit orbital model was found with an iterative nonlinear least-squares minimization scheme. The best-fit parameters are found in Table 4 . The reduced $\chi_{r}^{2}$ of the combined fit to PHASES, radial velocity, and previous differential astrometry data is 1.37. This combined set has 378 data points ( 49 of which are two-dimensional astrometric points) and 23 free parameters. The fits to the astrometric and radial velocity data for the various subsystems are shown in Figures 3-7. We find that the two shortperiod systems have eccentricities consistent with zero, and we therefore held these parameters fixed at zero for the fit. The time of maximum primary apparent velocity is chosen as zero orbital phase. To investigate the consistency of two astrometric data sets we also reran the fit, without including the previous astrometry. We find that the final results are consistent to within $1 \sigma$; including the previous astrometry does reduce certain parameter uncertainties by a small amount. In Table 4 we compare our best-fit values with those available in the literature (Abt \& Levy 1985; Balega et al. 1999) where possible; we find generally good agreement.

Finally, we considered the possibility of an additional massive body in the system. At this point, a full mass-period phase space search for a fifth component is computationally prohibitive and beyond the scope of this investigation. However, through the course of the investigation, we discovered a possible periodicity that warranted follow-up. Before we had determined the 7.89 day period of the Ab1-Ab2 system, we had searched the PHASES data for this third orbit signal with the period-searching program used to find the signatures of additional companions (Muterspaugh et al. 2006c); because the Aa-Ab and Aa1-Aa2 periods were known already, these orbits were seeded at their best-fit double Kepler values. While radial velocities eventually isolated the period for $\mathrm{Ab} 1-\mathrm{Ab} 2$ as 7.89 days, this astrometric search additionally identified a curious improvement near 56 days. The size of that orbit could have corresponded either to a brown dwarf or to a nearly equal luminosity star-star subsystem. Inspired by this identification, a five-component, quadruple-Keplerian model was seeded with the three known orbits (Aa-Ab, Aa1-Aa2, and Ab1-Ab2) and a fourth perturbation with a period of 56 days. Separately, the harmonic of 28 days was also investigated. This model included all astrometric and radial velocity data. The five-component model with a candidate period near 28 days $(\mathrm{C} / \mathrm{Ab}$ mass ratio $\sim 0.044)$ yields a significant improvement in the reduced $\chi^{2}$ of the fit over a four-body model (491 for 30 parameters and 397 degrees of freedom vs. 547 for 23 parameters and 404 degrees of freedom, corresponding to a $\sim 2.3 \sigma$ improvement from the $\chi^{2}$ ratio test). The 

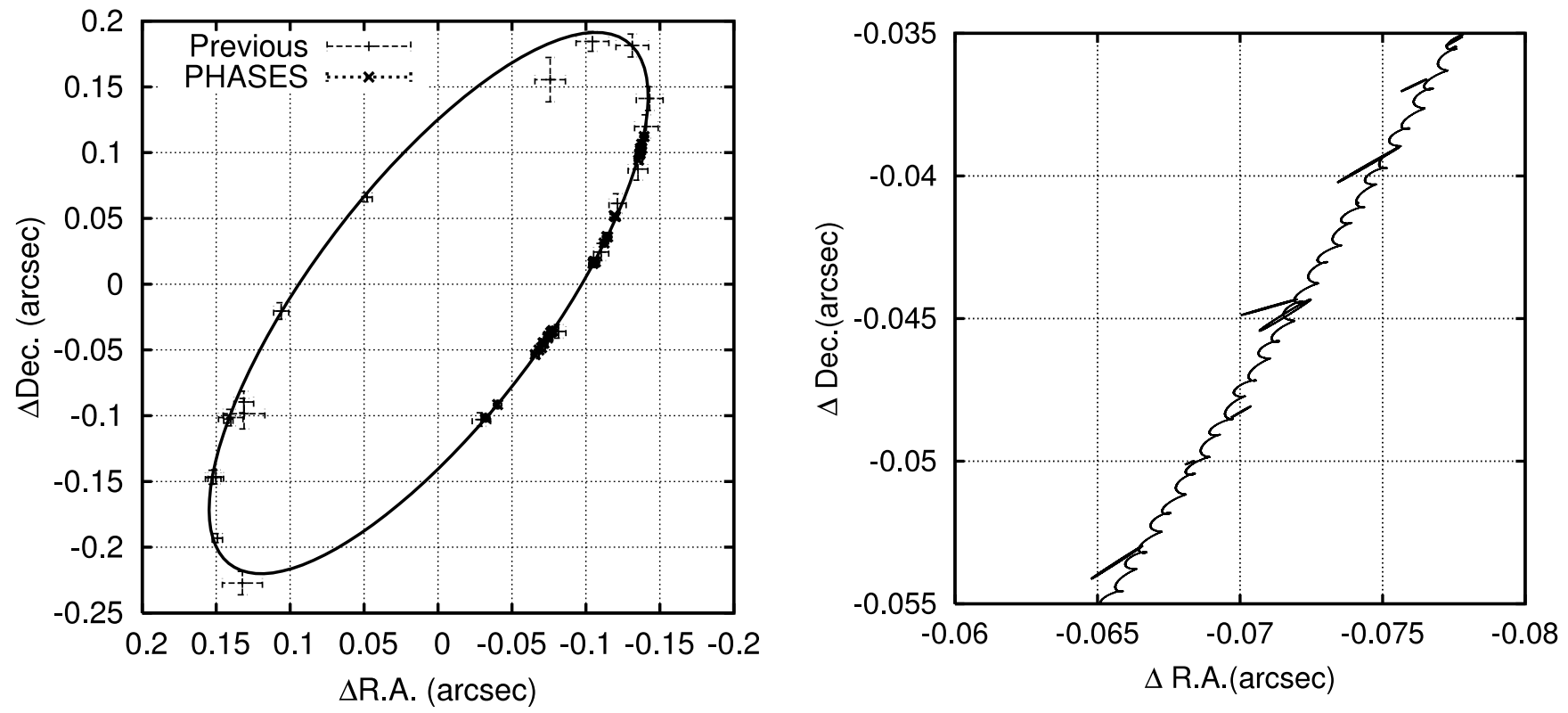

FIG. 3.- Left: Best-fit visual orbit of the 88 Tau Aa-Ab system, together with previously available astrometric data, and our PHASES astrometry. Note that the error

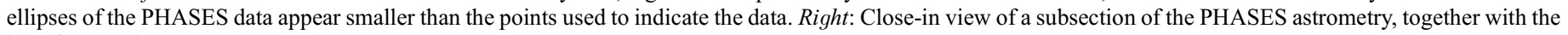
best-fit orbital model.

candidate period near 56 days yields a best-fit $\chi^{2} \sim 474$ for a $3.7 \sigma$ improvement over the four-component model, yet with a very large best-fit eccentricity $(0.93)$. However, we note that the 28 day period is close to the product of the $\mathrm{Aa}$ and $\mathrm{Ab}$ orbital periods ( 28.1 days) and the 56 day period is a harmonic of this, raising suspicion regarding the reliability of the solution. Hence the astrophysical significance of this additional component remains unclear. We do not claim this as a detection, but it is an intriguing result worthy of observational follow-up.

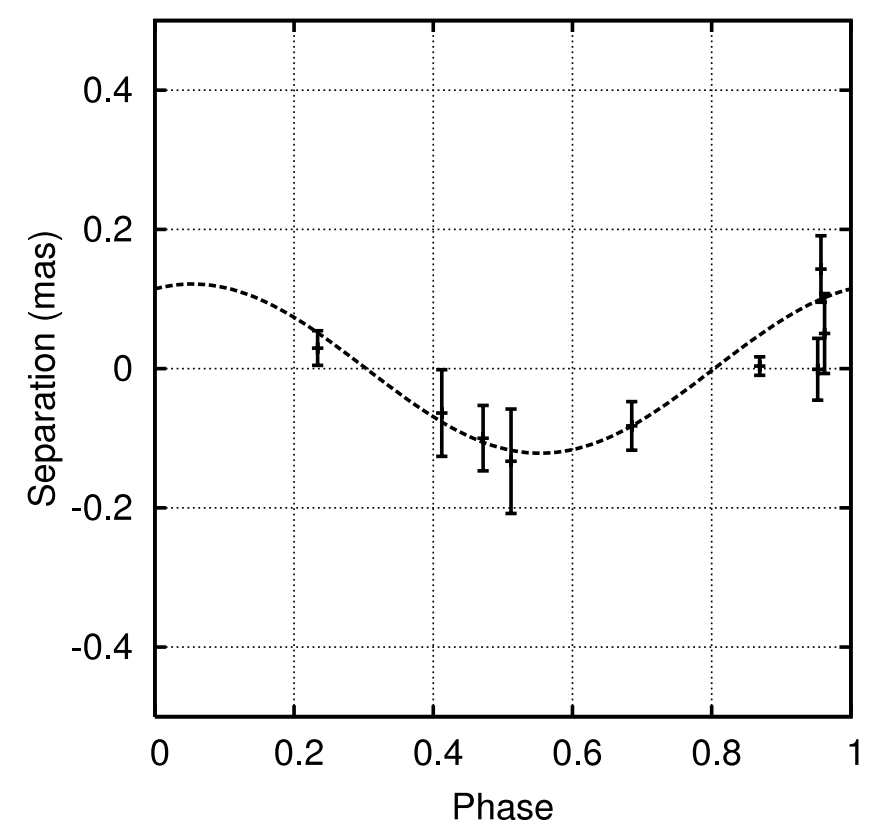

\subsection{Relative Orbital Inclinations}

The mutual inclination $\Phi$ of two orbits is given by

$$
\cos \Phi=\cos i_{1} \cos i_{2}+\sin i_{1} \sin i_{2} \cos \left(\Omega_{1}-\Omega_{2}\right),
$$

where $i_{1}$ and $i_{2}$ are the orbital inclinations and $\Omega_{1}$ and $\Omega_{2}$ are the longitudes of the ascending nodes. For this quadruple system we derive three separate mutual inclinations, corresponding to the three possible pairwise comparisons of the three orbits in this system

FIG. 4.-Astrometric orbit of the 88 Tau Aa1-Aa2 subsystem, projected along two different axes $\left(145^{\circ}\right.$ east of north on the left, $38^{\circ}$ on the right). In each case the

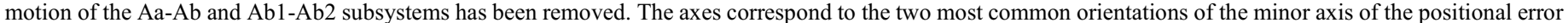

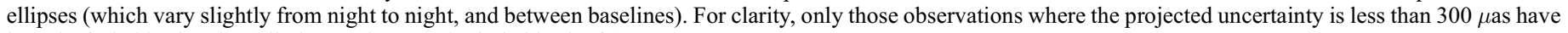
been included in the plot (all observations are included in the fit). 


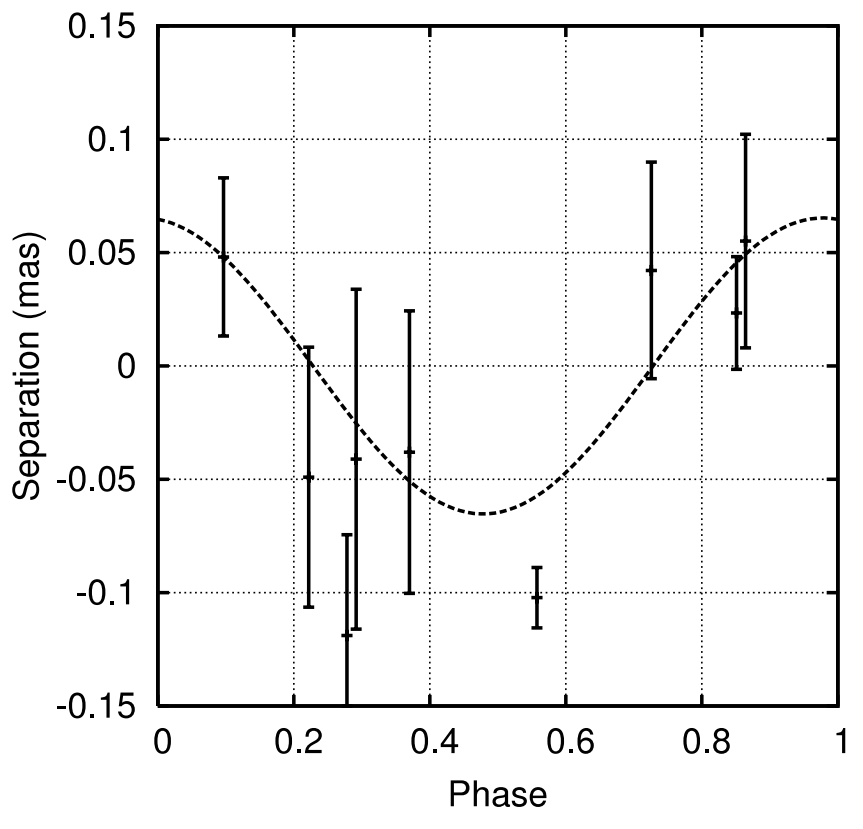

FIG. 5.-Astrometric orbit of the 88 Tau Ab1-Ab2 subsystem. The motion of the Aa-Ab and Aa1-Aa2 subsystems has been removed. The separations shown are projected along an axis oriented $145^{\circ}$ east of north, corresponding to mean orientation of the minor axis of the positional error ellipses (which vary slightly from night to night). For clarity, only those observations where the projected uncertainty is less than $300 \mu$ as have been included in the plot (all observations are included in the fit).

(i.e., Aab-Aa1Aa2, Aab-Ab1Ab2, and Aa1Aa2-Ab1Ab2). The resulting values found from our combined orbital solutions are given in Table 5. The large mutual inclinations indicate that the system orbits are not coplanar.

It should be noted that even with both COL-astrometry and radial-velocity data, there exists a parameter degeneracy corresponding to an exchange of the ascending and descending nodes together with a change in the luminosity ratio (interchanging which is the brighter star). Given one solution for the mass and luminosity

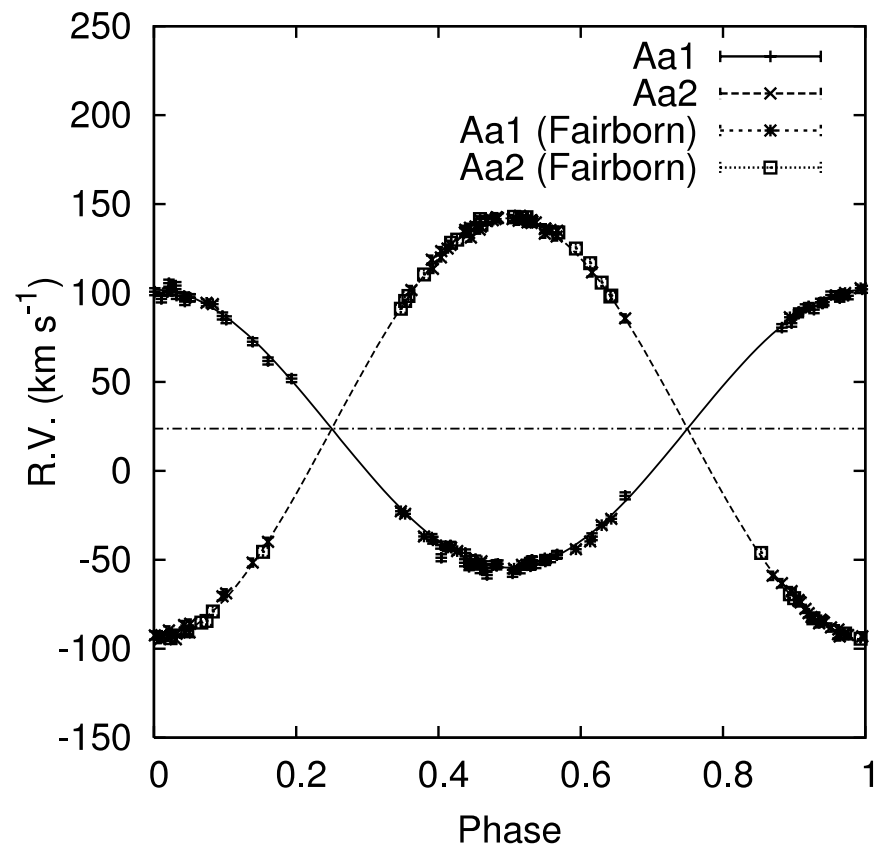

ratios ( $R$ and $L_{1}$ ), the other possible luminosity ratio can be found from

$$
L_{2}=\frac{2 R+R L_{1}-L_{1}}{1+2 L_{1}-R} .
$$

In a quadruple stellar system such as 88 Tau A there are four possible model solutions. However, as can be seen in Figure 2 the luminosity of the Aa1 component is clearly greater than the Aa2 component; hence we choose the solution where $L_{\mathrm{Aa} 1 \mathrm{Aa} 2}=0.24$. However, given the nearly equal masses of the Ab1 and Ab2 components, it is not entirely clear which is the preferred solution $\left(L_{\mathrm{Ab} 1 \mathrm{Ab} 2}=0.87\right.$ or 1.10$)$, and we calculate the two possible values for the corresponding mutual inclinations.

The two possible mutual inclination values of the $\mathrm{Ab}$ system are both in the range for inclination-eccentricity oscillations of $39.2^{\circ}-140.8^{\circ}$ (Kozai 1962), while the Aa system is not (although only $1 \sigma$ away from the limit). During these "Kozai cycles" the orbital eccentricity varies on a timescale $\propto P_{\text {out }}^{2} / P_{\text {in }}\left(\sim 10^{4} \mathrm{yr}\right.$ for the Ab system; Kiseleva-Eggleton \& Eggleton 2001), and in the absence of damping factors the eccentricity values would range from $\sim 0$ to $\sim 0.98$ (Kiseleva et al. 1998; however, see KiselevaEggleton \& Eggleton 2001 for a discussion of various factors that may limit these excursions). A full treatment of the dynamics of this system, including the effects of tidal friction, quadrupolar distortion, and general relativity, is beyond the scope of this paper.

Recently, Fabrycky \& Tremaine (2007) studied the effect that Kozai oscillations would have on the distributions of orbital properties of triple systems. Systems with mutual inclinations in the range where Kozai cycles occur evolve rapidly as tidal dissipation during the high-eccentricity (and hence close approach) phase of the oscillation causes the orbit of the inner binary to shrink. The resulting mutual inclination distribution is strongly peaked near the critical values of $39^{\circ}$ and $141^{\circ}$. It is therefore interesting to note that the Aa system is so close to one of these predicted values. The Ab system is more ambiguous: the preferred (albeit only weakly) solution for the Ab system yields a mutual inclination that would be expected to result in rapid orbital evolution.

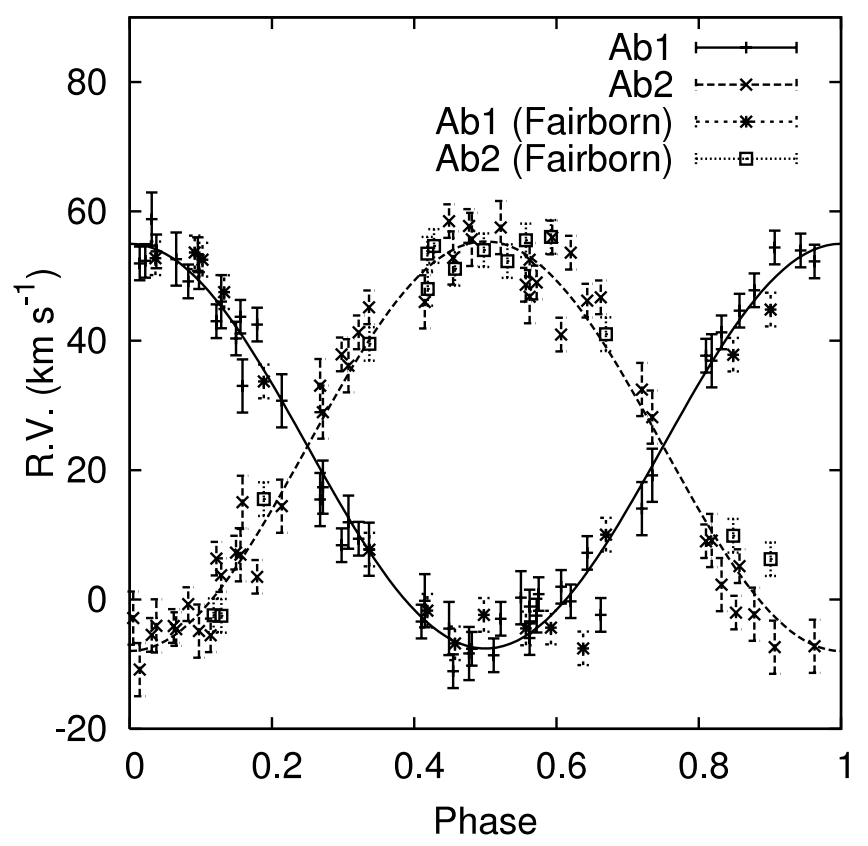

FIG. 6. - Left: Measured and model radial velocities of the Aa1-Aa2 subsystem, phased about the best-fit orbital model, and with the motions due to the Aa-Ab orbit removed. Right: Measured and model radial velocities of the Ab1-Ab2 subsystem, with the Aa-Ab motion removed. 


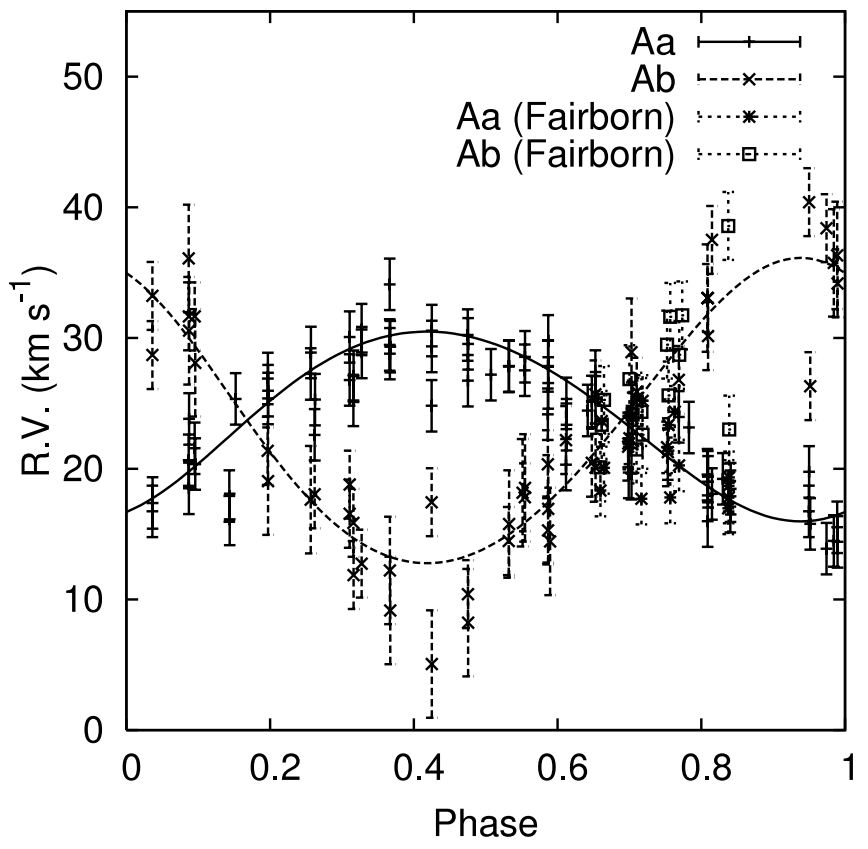

FIG. 7.- Measured and modeled radial velocities of the 88 Tau Aa-Ab system, with the motion due to the Aa1-Aa2 and Ab1-Ab2 systems subtracted.

Interestingly, the second possible solution is also relatively close to the critical limit. Clearly, further observations will be needed in order to remove the ambiguity in the node and hence mutual inclination.

\subsection{Component Masses and Distance}

This study represents the first determination of the orbital inclinations in this system, and hence the first time the masses have been determined; the precision achieved is $\sim 5 \%$ for the Aa components and $\sim 6 \%$ for the $\mathrm{Ab}$ components. The parallax is found to be $19.73 \pm 0.34$ mas $(1.7 \%$ uncertainty), placing the system at a greater distance than that estimated by Hipparcos $(21.68 \pm$ 0.82 mas; Perryman et al. 1997). Our greater distance resolves the mass/luminosity discrepancy pointed out by Balega et al. (1999) that arises if one assumes the Hipparcos distance to this system.

Schoeller et al. (1998) and Balega et al. (1999) stated that the 3.57 day binary is eclipsing but gave no reference for this claim. Our inclination for that binary is $110.6^{\circ}$, a value that is about $10^{\circ}$ above the maximum value required for eclipses to occur if canonical values are assumed for the radii of the two stars (Gray 1992). Thus, the system is not eclipsing. We also note that because of our lower inclination, our masses are about $0.2 M_{\odot}$ larger than those adopted by Balega et al. (1999).

\subsection{Component Luminosities}

As part of the combined astrometric and radial velocity fit we can solve for the $K$-band luminosity ratios of the components; this is because the distance and subsystem total masses are essentially determined by the observations of the wide Aa-Ab system, while the subsystem mass ratios are found from the subsystem radial velocities; this leaves only the component luminosity ratios dependent on the size of the observed astrometric perturbation.

While PTI cannot provide precise determinations of the total system magnitude $m_{K}$ or the Aa-Ab system differential magnitude $\Delta m_{K}$, these can be found in the literature. Balega et al. (2001) gave $\Delta m_{K^{\prime}}=1.29 \pm 0.12$ for the Aa-Ab system. We derive the resulting absolute $K$ magnitudes and list them in Table 5; the re-
TABLE 5

Derived System Parameters for 88 Tauri A

\begin{tabular}{|c|c|}
\hline Parameter & Value and Uncertainty \\
\hline 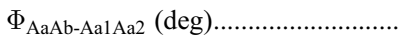 & $143.3 \pm 2.5$ \\
\hline 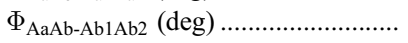 & $82.0^{\mathrm{a}} \pm 3.3$ \\
\hline 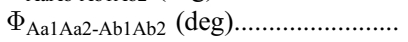 & $115.8^{\mathrm{b}} \pm 4.6$ \\
\hline$\pi(\operatorname{arcsec}) \ldots \ldots \ldots \ldots \ldots \ldots \ldots \ldots$ & $0.01973 \pm 0.00034$ \\
\hline$a_{\mathrm{AaAb}}(\mathrm{mas}) \ldots \ldots \ldots . . .$. & $240.1 \pm 5.3$ \\
\hline 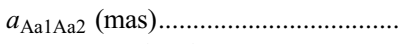 & $1.359 \pm 0.034$ \\
\hline 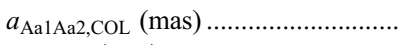 & $0.270 \pm 0.032$ \\
\hline$a_{\mathrm{Ab} 1 \mathrm{Ab} 2}(\mathrm{mas})$ & $1.967 \pm 0.054$ \\
\hline 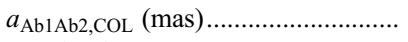 & $0.065 \pm 0.020$ \\
\hline 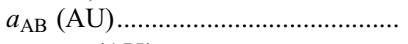 & $12.17 \pm 0.17$ \\
\hline 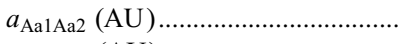 & $0.0689 \pm 0.0012$ \\
\hline$a_{\mathrm{Ab} 1 \mathrm{Ab} 2}(\mathrm{AU}) \ldots \ldots \ldots \ldots \ldots \ldots \ldots$ & $0.0997 \pm 0.0021$ \\
\hline 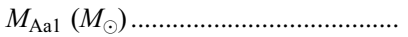 & $2.06 \pm 0.11$ \\
\hline 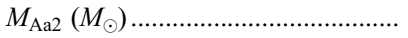 & $1.361 \pm 0.073$ \\
\hline 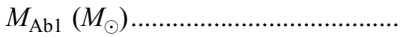 & $1.069 \pm 0.069$ \\
\hline 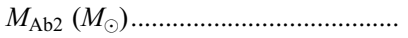 & $1.057 \pm 0.068$ \\
\hline$M_{K, \mathrm{Aal}} \ldots \ldots \ldots \ldots \ldots \ldots \ldots \ldots \ldots \ldots \ldots \ldots \ldots \ldots \ldots \ldots \ldots \ldots \ldots$ & $0.69 \pm 0.26$ \\
\hline 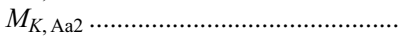 & $2.20 \pm 0.28$ \\
\hline 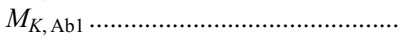 & $2.31 \pm 0.27$ \\
\hline 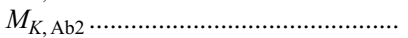 & $2.00 \pm 0.27$ \\
\hline
\end{tabular}

Notes.-Shown are the parameters derived from the best-fit model values in Table 4 and their uncertainties. COL refers to the amplitude of the motion of the center of light of the subsystem in question. Note that our combined astrometry and radial velocity model fits for the system masses directly.

a An alternate solution has $\Phi_{\mathrm{AaAb}-\mathrm{Ab} 1 \mathrm{Ab} 2}=58^{\circ}$ if $L_{\mathrm{Ab} 2} / L_{\mathrm{Ab} 1}>1$.

${ }^{\mathrm{b}}$ An alternate solution has $\Phi_{\mathrm{Aa} 1 \mathrm{Aa} 2-\mathrm{Ab} 1 \mathrm{Ab} 2}=107^{\circ}$ if $L_{\mathrm{Ab} 2} / L_{\mathrm{Ab} 1}>1$.

sults are dominated by the uncertainty in the total magnitude. Note that we assume the solution where $L_{\mathrm{Ab} 2} / L_{\mathrm{Ab} 1}<1$. We compare the determined masses and $K$-band absolute magnitudes to published theoretical isochrones (Girardi et al. 2002) in the range 0.7$2 \operatorname{Gyr}(Z=0.019)$. We find that the Aa components are consistent with the isochrones, but the Ab system components appear overluminous by $\sim 0.5 \mathrm{mag}$ (Fig. 8 ). However, this must be considered a preliminary finding since it is dependent on a single measurement of the Aa-Ab magnitude difference, and we have not taken into account the (presumably minor) effects of the slightly different bandpasses ( $K$ vs. $K^{\prime}$ ).

\subsection{Spectral Classes and $v \sin i$}

Strassmeier \& Fekel (1990) identified several luminositysensitive and temperature-sensitive line ratios in the $6430-6465 \AA$ region. Those critical line ratios and the general appearance of the spectrum were employed as spectral-type criteria. However, for stars that are hotter than about early G, the line ratios in the $6430 \AA$ region have little sensitivity to luminosity, so only the spectral class of an A or F star can be determined. The luminosity class is found by computing the absolute visual magnitude with the Hipparcos or our orbital parallax and comparing that magnitude to evolutionary tracks or a table of canonical values for giants and dwarfs.

The red-wavelength spectrum of 88 Tau was compared with those of slowly rotating Am, F, and G dwarfs. These reference stars, identified mostly from the lists of Abt \& Morrell (1995), Keenan \& McNeil (1989), and Fekel (1997) were observed at KPNO with the same telescope, spectrograph, and detector as our spectra of 88 Tau. With a computer program developed by Huenemoerder \& Barden (1984) and Barden (1985), various combinations of reference-star spectra were rotationally broadened, 


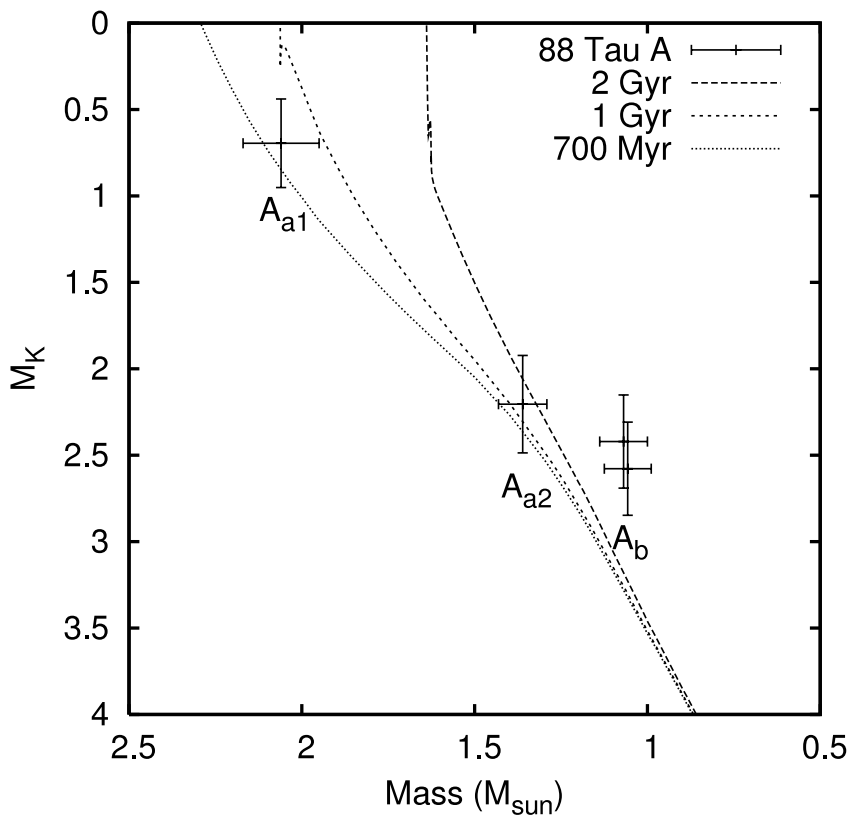

FIG. 8.-Mass vs. $K$ magnitude for the components of the 88 Tau A system, together with isochrones from Girardi et al. (2002).

shifted in radial velocity, appropriately weighted, and added together in an attempt to reproduce the spectrum of 88 Tau in the 6430 Å region. Abt \& Morrell (1995) classified 88 Tau as an Am star with spectral classes of A4, A6, and A7 for the calcium, hydrogen, and metal lines, respectively. Their classification of HR 3526 was identical to that of 88 Tau, so we adopted the spectrum of HR 3526 as the proxy for the primary of the 3.57 day binary, which dominates the spectrum at blue wavelengths and is still the strongest component in our red-wavelength region (Fig. 2). The three-subclass difference between the calcium $\mathrm{K}$ line type and metal line type indicates that this star is a marginal or mild Am star (Abt \& Bidelman 1969). A good fit to the lines of the 3.57 day secondary was produced by Procyon, spectral type F5 IV-V (Johnson \& Morgan 1953). Lines of the two components in the 7.89 day binary are similar in strength and rotation and were well represented by a spectrum of $70 \mathrm{Vir}$, spectral type G4 V (Keenan \& McNeil 1989). A fit with HR 483, spectral type G1.5 V (Keenan $\&$ McNeil 1989), used as a proxy for the 7.89 day binary pair, was nearly as good. Thus, the spectral classes of the four stars are A6m, F5, G2-3:, and G2-3:, where the colon indicates that the spectral class is more uncertain than usual because of the weakness of the lines. As shown by their positions in Figure 8, all four stars are dwarfs. The abundances of Procyon, 70 Vir, and HR 483 are essentially solar, indicating that the abundances of the components of 88 Tau, except for the Am star, are also close to solar.

The continuum intensity ratio of our best reference-star combination, fitted to the spectrum of $88 \mathrm{Tau}$, is $0.79: 0.11: 0.05: 0.05$. If we adopt the continuum intensity ratios as the luminosity ratios at $6430 \AA$, we obtain a magnitude difference of $2.1 \pm 0.3$ between the 3.57 day pair, and $2.4 \pm 0.3$ between the astrometric components, where the uncertainties are estimated. The $6430 \AA$ wavelength is about 0.6 of the way between the center of the Johnson $V$ and $R$ bandpasses.

With the procedure of Fekel (1997) we determined projected rotational velocities for the four components of 88 Tau from $10 \mathrm{KPNO}$ red-wavelength spectra. For each spectrum the full widths at half- maximum of two or three unblended lines in the $6430 \AA$ region were measured and the results averaged for each component. The instrumental broadening was removed, and the calibration polynomial of Fekel (1997) was used to convert the resulting broadening in angstroms into a total line broadening in $\mathrm{km} \mathrm{s}^{-1}$. From Fekel $(1997,2003)$ we assumed a macroturbulence of 0.0 for the Am star, $4 \mathrm{~km} \mathrm{~s}^{-1}$ for the mid-F star, and $3 \mathrm{~km} \mathrm{~s}^{-1}$ for the $\mathrm{G}$ stars. The resulting $v \sin i$ values are $37 \pm 2$ and $17 \pm 2 \mathrm{~km} \mathrm{~s}^{-1}$ for the primary and secondary of the 3.57 day binary, respectively, and $5 \pm 3 \mathrm{~km} \mathrm{~s}^{-1}$ for both components of the 7.89 day binary. Our value for the Aal component is consistent with the determination of $v \sin i=36 \mathrm{~km} \mathrm{~s}^{-1}$ by Royer et al. (2002).

To determine whether the rotational velocities of the binary components are synchronized, we assumed that the rotational axis of each component is parallel to its orbital axis. We then computed the equatorial rotational velocity for each component and compared it with its expected synchronous velocity, computed with its canonical radius (Gray 1992). The resulting equatorial rotational velocities are 40,18, 11, and $11 \mathrm{~km} \mathrm{~s}^{-1}$, for components Aa1, Aa2, $\mathrm{Ab} 1$, and $\mathrm{Ab} 2$, respectively. The computed synchronous rotational velocities are $24,19,6.5$, and $6.5 \mathrm{~km} \mathrm{~s}^{-1}$, respectively.

Component Aa1, the Am star and primary of the 3.57 day binary, is the only one of the four components that does not have a convective atmosphere. It is also the only component that is clearly not rotating synchronously: its equatorial rotational velocity is 1.67 times faster than synchronous. On the other hand its F5 companion, component Aa2, is rotating synchronously. Because of the relatively large uncertainties of our $v \sin i$ measurements for $\mathrm{Ab} 1$ and $\mathrm{Ab} 2$, it is possible that the two components of the 7.89 day are also synchronously rotating.

\section{CONCLUSION}

PHASES interferometric astrometry has been used together with radial velocity data to measure the orbital parameters of the quadruple star system 88 Tau A, and in particular to resolve the apparent orbital motion of the close Aa1-Aa2 and Ab1-Ab2 pairs. We have made the first determination of the period of the Ab binary system and found it to consist of a pair of nearly equal-mass $G$ stars. The amplitude of the Ab1-Ab2 center-of-light motion is only $\sim 65 \mu$ as, indicating the level of astrometric precision attainable with interferometric astrometry. We are able to resolve the orbital motion of all of the components, and hence determine the orbital inclinations and component masses with a precision of a few percent. Finally, we are able to determine the mutual inclinations of the various orbits.

We wish to acknowledge the extraordinary observational efforts of K. Rykoski. Observations with PTI are made possible thanks to the efforts of the PTI Collaboration, which we acknowledge. This research has made use of services from the Michelson Science Center, California Institute of Technology, http://msc.caltech.edu. Part of the work described in this paper was performed at the Jet Propulsion Laboratory under contract with the National Aeronautics and Space Administration. This research has made use of the SIMBAD database, operated at CDS, Strasbourg, France, and of data products from the Two Micron All Sky Survey, which is a joint project of the University of Massachusetts and the Infrared Processing and Analysis Center, California Institute of Technology, funded by NASA and the NSF. We thank T. Willmitch for measuring some of the early KPNO spectra. The work of F. C. F. and M. W. has been supported in part by NASA grant NCC5-511 and 
NSF grant HRD-9706268. PHASES is funded in part by the California Institute of Technology Astronomy Department, and by the National Aeronautics and Space Administration under grant NNG05GJ58G issued through the Terrestrial Planet Finder Foundation Science Program. This work was supported in part by the
National Science Foundation through grants AST 03-00096, AST 05-07590, and AST 00-5366. M. W. M. is grateful for the support of a Townes fellowship. M. K. is supported by NASA through grant NNG04GM62G and the Polish Ministry of Education and Science through grant 1P03D 02129.
Abt, H. A., \& Bidelman, W. P. 1969, ApJ, 158, 1091

Abt, H. A., \& Levy, S. G. 1985, ApJS, 59, 229

Abt, H. A., \& Morrell, N. I. 1995, ApJS, 99, 135

Balega, I. I., Balega, Y. Y., Hofmann, K.-H., Tokovinin, A. A., \& Weigelt, G. P. 1999, Astron. Lett., 25, 797

Balega, I. I., Balega, Y. Y., Hofmann, K.-H., \& Weigelt, G. 2001, Astron. Lett., 27,95

Barden, S. C. 1985, ApJ, 295, 162

Bopp, B. W., Evans, D. S., \& Laing, J. D. 1970, MNRAS, 147, 355

Burkhart, C., \& Coupry, M. F. 1988, A\&A, 200, 175

Colavita, M. M., et al. 1999, ApJ, 510, 505

Cowley, A., Cowley, C., Jaschek, M., \& Jaschek, C. 1969, AJ, 74, 375

Eaton, J. A., \& Williamson, M. H. 2004, Proc. SPIE, 5496, 710

Fabrycky, D., \& Tremaine, S. 2007, ApJ, 669, 1298

Fekel, F. C. 1997, PASP, 109, 514

1999, in IAU Colloq. 170, Precise Stellar Radial Velocities, ed. J. B.

Hearnshaw \& C. D. Scarfe (ASP Conf. Ser. 185; San Francisco: ASP), 378 2003, PASP, 115, 807

Fitzpatrick, M. J. 1993, in ASP Conf. Ser. 52, Astronomical Data Analysis Software and Systems II, ed. R. J. Hanisch, R. V. J. Brissenden, \& J. Barnes (San Francisco: ASP), 472

Girardi, L., Bertelli, G., Bressan, A., Chiosi, C., Groenewegen, M. A. T., Marigo, P., Salasnich, B., \& Weiss, A. 2002, A\&A, 391, 195

Gray, D. F. 1992, The Observation and Analysis of Stellar Photospheres (Cambridge: Cambridge Univ. Press)

Hartkopf, W. I., McAlister, H. A., \& Mason, B. D. 2001, AJ, 122, 3480

Huenemoerder, D. P., \& Barden, S. C. 1984, BAAS, 16, 510

Johnson, H. L., \& Morgan, W. W. 1953, ApJ, 117, 313

Keenan, P. C., \& McNeil, R. C. 1989, ApJS, 71, 245

Kiseleva, L. G., Eggleton, P. P., \& Mikkola, S. 1998, MNRAS, 300, 292

\section{REFERENCES}

Kiseleva-Eggleton, L., \& Eggleton, P. P. 2001, in ASP Conf. Ser. 229, Evolution of Binary and Multiple Star Systems, ed. Ph. Podsiadlowski et al. (San Francisco: ASP), 91

Kozai, Y. 1962, AJ, 67, 579

Lane, B. F., \& Muterspaugh, M. W. 2004, ApJ, 601, 1129

McAlister, H. A., Hartkopf, W. I., Hutter, D. J., \& Franz, O. G. 1987, AJ, 93 , 688

Moore, C. E., Minnaert, M. G. J., \& Houtgast, J. 1966, The Solar Spectrum $2935 \AA$ to $8770 \AA$ (NBS Monogr. 61; Washington: GPO)

Muterspaugh, M. W., Lane, B. F., Konacki, M., Burke, B. F., Colavita, M. M., Kulkarni, S. R., \& Shao, M. 2005, AJ, 130, 2866 . 2006a, A\&A, 446, 723

Muterspaugh, M. W., Lane, B. F., Konacki, M., Wiktorowicz, S., Burke, B. F., Colavita, M. M., Kulkarni, S. R., \& Shao, M. 2006b, ApJ, 636, 1020

Muterspaugh, M. W., Lane, B. F., Kulkarni, S. R., Burke, B. F., Colavita, M. M., \& Shao, M. 2006c, ApJ, 653, 1469

Perryman, M. A. C., et al. 1997, The Hipparcos and Tycho Catalogues (ESA SP-1200; Noordwijk: ESA)

Royer, F., Grenier, S., Baylac, M.-O., Gómez, A. E., \& Zorec, J. 2002, A\&A, 393, 897

Scarfe, C. D., Batten, A. H., \& Fletcher, J. M. 1990, Publ. Dominion Astrophys. Obs., 18, 21

Schoeller, M., Balega, I. I., Balega, Y. Y., Hofmann, K.-H., Reinheimer, T., \& Weigelt, G. 1998, Astron. Lett., 24, 283

Skrutskie, M. F., et al. 2006, AJ, 131, 1163

Sterzik, M. F., \& Tokovinin, A. A. 2002, A\&A, 384, 1030

Strassmeier, K. G., \& Fekel, F. C. 1990, A\&A, 230, 389

Tokovinin, A. A. 1997, A\&AS, 124, 75

Tokovinin, A. A., \& Gorynya, N. A. 2001, A\&A, 374, 227

Torres, G., \& Ribas, I. 2002, ApJ, 567, 1140 